\title{
\begin{tabular}{l|l} 
Mibraries & DSpace@MIT
\end{tabular}
}

\author{
MIT Open Access Articles
}

\section{Large-Area Atom Interferometry with Frequency-Swept Raman Adiabatic Passage}

The MIT Faculty has made this article openly available. Please share how this access benefits you. Your story matters.

Citation: Kotru, Krish, David L. Butts, Joseph M. Kinast, and Richard E. Stoner. "Large-Area Atom Interferometry with Frequency-Swept Raman Adiabatic Passage." Phys. Rev. Lett. 115, 103001 (August 2015). ( 2015 American Physical Society

As Published: http://dx.doi.org/10.1103/PhysRevLett.115.103001

Publisher: American Physical Society

Persistent URL: http://hdl.handle.net/1721.1/98300

Version: Final published version: final published article, as it appeared in a journal, conference proceedings, or other formally published context

Terms of Use: Article is made available in accordance with the publisher's policy and may be subject to US copyright law. Please refer to the publisher's site for terms of use. 


\title{
Large-Area Atom Interferometry with Frequency-Swept Raman Adiabatic Passage
}

\author{
Krish Kotru, ${ }^{1,2}$ David L. Butts, ${ }^{2}$ Joseph M. Kinast, ${ }^{2}$ and Richard E. Stoner ${ }^{2}$ \\ ${ }^{1}$ Department of Aeronautics and Astronautics, Massachusetts Institute of Technology, Cambridge, Massachusetts 02139, USA \\ ${ }^{2}$ The C. S. Draper Laboratory, Cambridge, Massachusetts 02139, USA
}

(Received 27 January 2015; published 31 August 2015)

\begin{abstract}
We demonstrate light-pulse atom interferometry with large-momentum-transfer atom optics based on stimulated Raman transitions and frequency-swept adiabatic rapid passage. Our atom optics have produced momentum splittings of up to 30 photon recoil momenta in an acceleration-sensitive interferometer for laser cooled atoms. We experimentally verify the enhancement of phase shift per unit acceleration and characterize interferometer contrast loss. By forgoing evaporative cooling and velocity selection, this method lowers the atom shot-noise-limited measurement uncertainty and enables large-area atom interferometry at higher data rates.
\end{abstract}

DOI: 10.1103/PhysRevLett.115.103001

PACS numbers: 37.25.+k, 03.75.Be, 03.75.Dg

Light-pulse atom interferometry (LPAI) is a preeminent method for precision measurements of inertial forces $[1,2]$ and fundamental physical constants [3,4]. Highly sensitive LPAI systems may be an enabling technology for nextgeneration inertial navigators [5-7], gravitational wave detectors [8], and tests of the equivalence principle [9]. Nevertheless, many light-pulse atom interferometers are presently limited by atom beam splitters and mirrors that create small momentum separations (two photon recoil momenta) between diffracting wave packets. The sensitivity of these interferometers typically increases with the effective area enclosed by the interfering wave packets [10]. Since this area is proportional to momentum separation, sensitivity can be enhanced using atom optics that generate large momentum transfer (LMT). Previous demonstrations of atom interferometry with LMT atom optics have taken several approaches, including sequential application of stimulated Raman transitions [11,12], Raman composite pulses [13], and stimulated Raman adiabatic rapid passage (STIRAP) pulses [14], as well as application of multiphoton-Bragg transitions [15-17], and Bloch oscillations in an optical lattice $[18,19]$.

In most of these demonstrations, cold atoms from a magneto-optical trap (MOT) were either evaporatively cooled or velocity selected-both of which typically discard $>90 \%$ of the original atom sample. A reduced atom number is detrimental to atom shot-noise-limited measurement uncertainty and to operation at fast data rates. A slower data rate results because, following every measurement cycle, the steady-state atom number in the MOT must be recovered primarily from roomtemperature atoms. When cold atoms are recaptured, however, fewer atoms must be loaded from the roomtemperature background vapor, thus allowing the data rate to be increased above $100 \mathrm{~Hz}$ [20]. High data rates are crucial for atom interferometric measurements of dynamic signals, such as rapidly varying accelerations and rotations of moving platforms, as well as strains from high frequency $(\sim 10 \mathrm{~Hz})$ gravitational waves $[8,19]$. The fastest data rates with evaporative cooling have been limited to $\leq 1.3 \mathrm{~Hz}$ [21]; velocity selection at high data rates requires the added complexity of a $2 \mathrm{D}$ MOT to maintain atom number [22].

In this Letter, we demonstrate cold atom interferometers with up to $30 \hbar k$ beam splitter pulses, while forgoing evaporative cooling and velocity selection. The atom beam splitters are implemented in an acceleration-sensitive interferometer and use a combination of stimulated Raman transitions and frequency-swept adiabatic rapid passage (ARP). These atom optics will enable large-area atom interferometry with improved counting statistics, fast data rates, and reduced constraints on the atom temperature. Our approach to ARP fundamentally and practically differs from the demonstration of STIRAP in Ref. [14], as will be discussed below.

We apply LMT atom optics in a Mach-Zehnder atom interferometer [23], depicted in Fig. 1(a). The interferometer is composed of a beam splitter pulse sequence that divides the atom wave packet, a mirror sequence that brings the wave packets back together, and a second beam splitter sequence that overlaps the wave packets to create interference [11]. The first $(\pi / 2)$, middle $(\pi)$, and final $(\pi / 2)$ pulses drive Raman transitions that produce the nominal interferometer with $2 \hbar k$ momentum splitting between diffracting wave packets. Achieving higher momentum splittings requires "augmentation" pulses with wave vectors orientated according to the vertical arrows in Fig. 1(a). In the absence of gravity gradients, this pulse sequence produces a relative phase between the interferometer arms given by the expression $\Delta \phi=\mathbf{k}_{\text {eff }} \cdot \mathbf{a}\left[(2 N+1) T^{2}-2 N(N+1) T \tau\right]$ [11], where $\mathbf{a}$ is an acceleration, $\mathbf{k}_{\text {eff }}$ is the effective Raman wave vector, $T$ is the dwell time, and augmentation pulses in the beam splitter are numbered 1 to $N$ and separated by time $\tau$. 
(a)

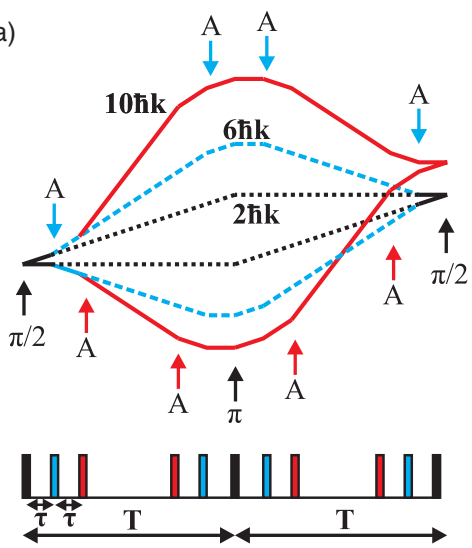

(b)

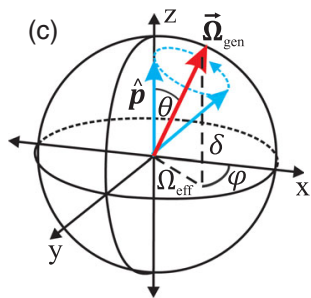

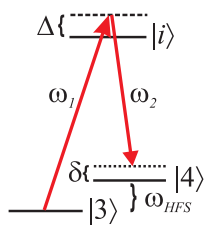

FIG. 1 (color online). (a) Diagram of pulse timings and wavepacket trajectories for Mach-Zehnder interferometers with $2 \hbar k$, $6 \hbar k$, and $10 \hbar k$ atom optics. Augmentation pulses (A) are either Raman $\pi$ or ARP pulses. (b) Stimulated Raman transition coupling the ground states in a $\Lambda$ system. (c) Bloch sphere depiction of frequency-swept adiabatic rapid passage, with poles corresponding to alkali-metal clock states.

As shown in the energy-level diagram in Fig. 1(b), each atom optics pulse generates a two-photon Raman transition between alkali-metal hyperfine ground states $|3\rangle$ and $|4\rangle$, separated by energy $\hbar \omega_{\mathrm{HFS}}$. The transition is produced by counterpropagating optical frequencies $\omega_{1}$ and $\omega_{2}$, with two-photon detuning $\delta$ and one-photon detuning $\Delta$, defined with respect to an excited state $|i\rangle$. To improve the atomic coherence during LMT, we use augmentation pulses based on frequency-swept ARP with Raman transitions [24]. In direct analogy to ARP methods from nuclear magnetic resonance, Raman ARP in an effective two-level system inverts the population with high fidelity by slowly sweeping the Raman detuning $\delta$ through resonance $[25,26]$. As seen on the Bloch sphere in Fig. 1(c), the Bloch vector $\hat{\mathbf{p}}$ adiabatically follows the Raman drive field $\vec{\Omega}_{\text {gen }}$ when $\dot{\theta} \ll \Omega_{\text {gen }}$. Here, $\Omega_{\text {gen }} \equiv \sqrt{\delta^{2}+\Omega_{\text {eff }}^{2}}$ determines the rate of precession of $\hat{\mathbf{p}}$ about $\vec{\Omega}_{\text {gen }}$, and $\Omega_{\text {eff }}$ is the magnitude of the two-photon Rabi rate. We control $\theta$ by varying $\delta$ and $\Omega_{\text {eff }}$.

We emphasize that interferometry with adiabatic transfer based on STIRAP [14] fundamentally differs from the frequency-swept ARP approach used here, as STIRAP relies solely on optical intensity modulation with $\delta=0$ and $\Delta \approx 0$ [27]. In practical terms, STIRAP is ill-suited to our interferometer, since it would cause half the atoms to spontaneously emit during each augmentation pulse and significantly decrease sensitivity [23].

In the adiabatic limit, frequency-swept ARP imprints a dynamic phase $\gamma= \pm \int d t \Omega_{\text {gen }}(t) / 2$ onto the atom wave packet with a sign that depends on the internal state label [26]. Since augmentation pulses act simultaneously on both internal states - one in each intereferometer arm-every ARP adds a phase with magnitude $|2 \gamma|$ to the interferometer output. The dependence of $\gamma$ on optical intensity can create decoherence when the spatial intensity pattern varies across the atom sample. Our interferometer, however, maintains coherence because pairs of identical ARPs nearly cancel the dynamic phase imprinted on a particular wave packet. This cancellation, or "rephasing," is efficient when beam splitter augmentation pulses occur in rapid succession. A cold atom in this case traverses just a few microns between pulses and thus avoids large-scale spatial intensity variation. A quantitative evaluation of dynamic phase is provided below, as part of the discussion of experimental interferometry results.

To produce laser cooled atom samples, we used the apparatus described in Ref. [24]. Each measurement began with the loading of $\sim 10^{6}{ }^{133} \mathrm{Cs}$ atoms into a MOT. Polarization gradient cooling reduced the sample temperature to $9 \mu \mathrm{K}$. Prior to interferometry, about $90 \%$ of the atoms were optically pumped to the $\mid 6^{2} S_{1 / 2}, F=4, m_{F}=$ $0\rangle$ upper clock state and then transferred to the $\left|6^{2} S_{1 / 2}, F=3, m_{F}=0\right\rangle$ lower clock state with a microwave $\pi$ pulse. A pusher beam removed the remaining $10 \%$ of atoms from the interaction region. Following the interferometer pulse sequence, we measured the atom population in each clock state by sampling the laser induced fluorescence $f_{3}, f_{4}$ from each hyperfine ground state with a photodiode. The interferometer phase was then extracted from the normalized $F=4$ population, $f_{4} /\left(f_{3}+f_{4}\right)$.

Two injection-locked Fabry-Perot slave lasers produced Raman frequencies $\omega_{1}$ and $\omega_{2}$. Both lasers were seeded by a master external cavity diode laser, whose output was phase modulated by an electro-optic modulator (EOM) driven at $\sim 9 \mathrm{GHz}$. The EOM produced optical frequency sidebands spaced about the carrier by integer multiples of the driving frequency. Each slave laser received roughly $100 \mu \mathrm{W}$ of optical power from the phase-modulated master and was tuned to predominantly amplify either the carrier or the negative first-order frequency sideband. In addition, the master laser was red detuned from the $\mid 6^{2} S_{1 / 2}, F=$ $3\rangle \rightarrow\left|6^{2} P_{3 / 2}, F=4\right\rangle$ transition by $-3.9 \mathrm{GHz}$ to reduce spontaneous emission. The two Raman frequencies were delivered to the vacuum cell with separate polarization-maintaining optical fibers. The fiber outputs were collimated to $1 / e^{2}$ intensity diameters of $7.1 \mathrm{~mm}$, were crossed-linearly polarized with 1 " polarization beamsplitting cubes, and counterpropagated along a common axis that was aligned to within $\pm 0.5^{\circ}$ of vertical.

Agile control of the Raman detuning was achieved through the rf signal delivered to the EOM. The rf was produced by mixing the $30-\mathrm{MHz}$ output of a 625 megasamples/s arbitrary waveform generator with a constant $\sim 9-\mathrm{GHz}$ signal using a single-sideband mixer. During an interferometer sequence, the Raman frequency difference was chirped at $\pm 23 \mathrm{kHz} / \mathrm{ms}$ to continually match the Doppler-shifted Raman resonance in a $1 \mathrm{~g}$ environment. The upward or downward orientation of $\mathbf{k}_{\text {eff }}$ determined the sign of the chirp rate. A combination 
of two AOMs and polarization-selective optics enabled rapid optical switching and reversal of $\mathbf{k}_{\text {eff }}$. We increased the optical power in each Raman beam to $\sim 100 \mathrm{~mW}$ with tapered amplifier diodes. The resulting two-photon Rabi rate was $\Omega_{\text {eff }}=2 \pi \times 200 \mathrm{kHz}$, corresponding to a Raman $\pi$ pulse duration of $t_{\pi}=2.5 \mu \mathrm{s}$. The tapered amplifier drive currents were also tuned to obtain a ratio of optical powers that canceled the differential AC Stark shift of the clock states.

To reduce spontaneous emission during LMT, we used the relatively short tan/tanh ARP pulse [24,28-30]. The time-dependent ARP detuning was $\delta(t)=$ $\Omega_{\mathrm{ARP}} \tan \left[\alpha\left(2 t / T_{\pi}-1\right)\right]$, where $t \in\left\{0, T_{\pi}\right\}, T_{\pi}$ sets the total sweep duration, $\Omega_{\mathrm{ARP}}$ alters the sweep rate, and $\alpha=\arctan \left(\delta_{\max } / \Omega_{\mathrm{ARP}}\right)$, with $\delta_{\max }$ being the maximum detuning. The optical intensity was proportional to $\tanh \left[7.5\left(1-\left|2 t / T_{\pi}-1\right|\right)\right]$. In our apparatus, a $\tan / \tanh$ pulse with duration $T_{\pi}=10 t_{\pi}$ achieved $\sim 96 \%$ transfer efficiency over a broad range of detunings and was limited primarily by spontaneous emission.

To verify the enhancement of phase shift per unit acceleration $\Delta \phi / a$, we produced interferograms while varying the chirp rate of the Raman frequency difference. A short dwell time of $T=1 \mathrm{~ms}$ was used to reduce phase noise from environmental vibration. Other experimental parameters included $T_{\pi}=3 t_{\pi}, \quad \Omega_{\mathrm{eff}}=\Omega_{\mathrm{ARP}}=$ $2 \pi \times 200 \mathrm{kHz}, \delta_{\max }=2 \pi \times 15 \mathrm{MHz}$, and $\tau=41 \mu \mathrm{s}$. In response to chirp rate variation, interferograms with up to $30 \hbar k$ beam splitter pulses exhibited periods that decreased with higher LMT order and matched expected values, as shown in Fig. 2. Similar agreement was verified for up to

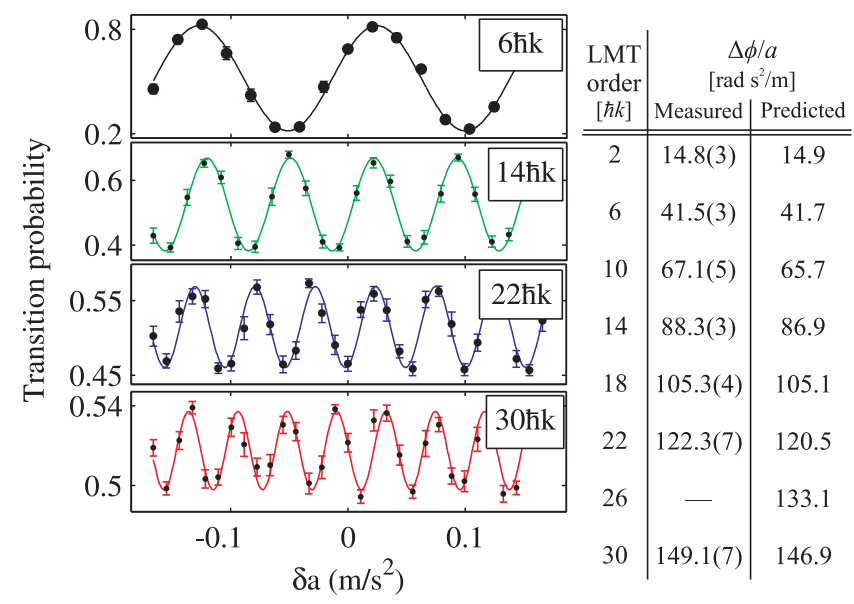

FIG. 2 (color online). Phase shift per unit acceleration $\Delta \phi / a$ for various LMT orders. Interferograms were acquired by perturbing the chirp rate of the Raman difference frequency. The resulting Doppler shift mimics a relative acceleration $\delta a$ between the atoms and Raman beams. Points represent 10- or 15-shot averages, error bars indicate standard error, and lines are fitted sine waves. The table shows reasonable agreement between measured and predicted values ( $26 \hbar k$ was not measured).
$14 \hbar k$ beam splitters using Raman $\pi$ pulses and tan/tanh pulses with $T_{\pi}=5 t_{\pi}$.

While the LMT interferometers clearly enhanced the phase shift per unit acceleration, the contrast $C$ of the interferograms (i.e., the peak-to-peak variation in transition probability) was simultaneously degraded. Maintaining contrast is important because it scales the measurement signal-to-noise ratio. To eliminate systematic underestimation of contrast due to vibration-induced phase noise, we assessed $C$ using histograms of transition probability measurements from each LMT interferometer. The arcsine probability density function characterizes the statistics of the transition probability in the limit of uniform random phase noise $[6,16]$. In our experiments, this limit was effectively produced through a combination of vibrationdriven phase noise and deliberate variation of the interferometer phase [31], as seen in Fig. 3(a). We therefore fit the arcsine distribution to our histograms, while keeping $C$ a free parameter. The resulting contrast estimates are shown in Fig. 3(b). At all LMT orders, tan/tanh interferometers achieve higher contrast than interferometers based on Raman $\pi$ pulses. The observed loss of contrast with increasing LMT order is similar to results from Ref. [15] and compares favorably to results from Refs. $[11,13,18]$. Using velocity-selected samples with temperatures of $\sim 100 \mathrm{nK}$ along the Raman beam axis, we observed a nominal increase in contrast, suggesting that inhomogeneity in the temperature-dependent Doppler detuning was not a dominant loss mechanism.

The loss of contrast observed with increasing LMT order was driven by several factors: single-photon excitations;

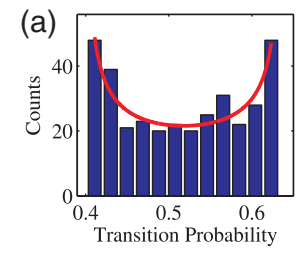

(c)
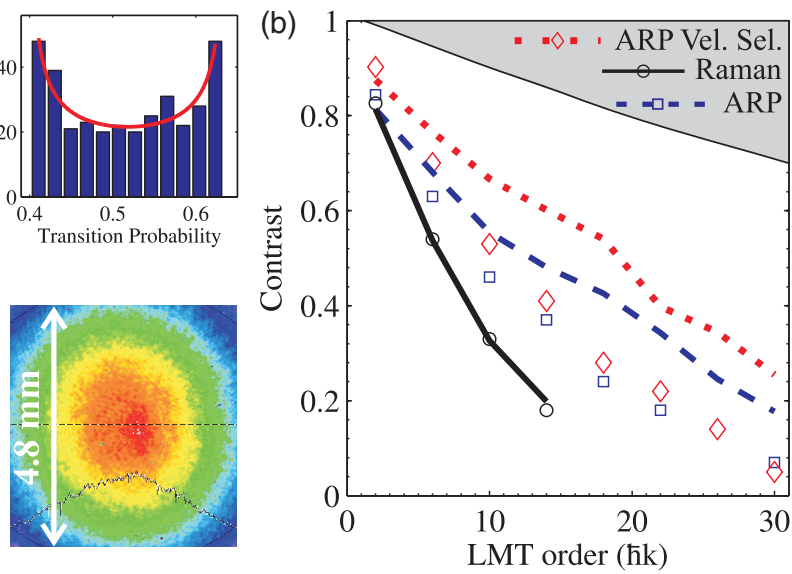

FIG. 3 (color online). (a) Histogram of transition probabilities from an $18 \hbar k$ interferometer with fitted probability density function (red curve). (b) Contrast as a function of LMT order. Lines are Monte Carlo predictions, points are contrast fits to histograms [see (a)], and fit uncertainties are smaller than symbol sizes. For tan/tanh interferometers, spontaneous emission-limited contrast corresponds to the bottom of the grey region. (c) Spatial intensity profile of the central portion of one Raman beam. 
detuning offsets due to the oppositely directed recoil velocities of each interferometer arm; detuning inhomogeneity due to temperature-dependent Doppler shifts; and Rabi rate and spatial phase inhomogeneity due to a combination of optical wave front distortion and thermal motion of the atoms. To jointly study these effects, we used Monte Carlo interferometer simulations. The inhomogeneity effects listed above were accounted for by using normally distributed initial atom positions and velocities. The Raman process was modeled as an effective two-level system [32], and we assumed uniform Raman wave fronts. To reduce computation times, probability amplitudes corresponding to population loss were not computed. A CCD image of the spatial intensity profile of only one Raman laser beam, shown in Fig. 3(c), was used to approximate the true variation in $\Omega_{\text {eff }}$ with position. To account for spontaneous emission, we scaled the simulated contrast results by $\left(1-R_{\text {sd }}\right)^{4 N t_{\text {aug }}+2}$, where $t_{\text {aug }}$ was the augmentation pulse duration in units of $t_{\pi}$, and $R_{\mathrm{sd}}=0.004$ was the population fraction lost to spontaneous decay per $\pi$ pulse.

Using known experimental parameters and adjustments to the atom sample size (order 10\%), the simulation produced contrast values that agreed with measurements based on Raman $\pi$ augmentations, as shown in Fig. 3(b). With the same experimental parameters, simulations based on tan/tanh augmentation pulses predicted higher levels of contrast at all LMT orders. These predictions were borne out qualitatively in experiments, though the measured contrast values were lower. The discrepancy may have resulted from dephasing due to aberrations in the true Raman beam wave front, as well as an optimistic model of the Raman beam intensity profile, which accounted for just one of the two Raman beams.

As a function of dwell time $T$, the measured contrast [Fig. 4(a)] decreased at a rate of roughly $0.03 / \mathrm{ms}$ for all LMT orders. This trend was likely due to the transverse motion of atoms in laser beams with spatially nonuniform intensity and wave front aberrations, both of which increasingly dephase the atoms over longer $T$ [19]. The transverse $\sim 2-\mathrm{cm} / \mathrm{s}$ RMS velocity of a $9-\mu \mathrm{K}$ cloud of ${ }^{133} \mathrm{Cs}$ atoms, with an initial $1 / e^{2}$ diameter of $2 \mathrm{~mm}$, causes a $40 \%$ expansion of the sample during an interferometer with $T=8.5 \mathrm{~ms}$. This expansion can be reduced by roughly a factor of 3 through improved laser cooling [15]—still without evaporative cooling or velocity selection.

Contrast sensitivity to ARP dynamic phase $\gamma$ was assessed by varying the ARP pulse duration $T_{\pi}$, because the uncertainty in $\gamma$ due to intensity inhomogeneity is proportional to $T_{\pi}$. The contrast as a function of increasing $T_{\pi}$, seen in Fig. 4(b), decreased at all LMT orders, even though the velocity acceptance of the ARP pulses improved with duration. Both spontaneous emission and dephasing of $\gamma$ contributed to this trend. However, Monte Carlo simulations with ideal Gaussian laser beams, ARP pulses with $T_{\pi}=10 t_{\pi}$, and even values of $N$ predicted nearly
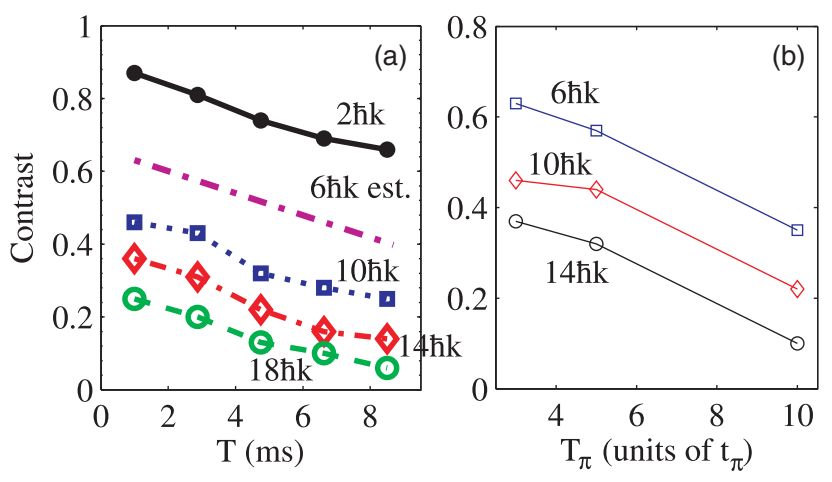

FIG. 4 (color online). (a) Contrast as a function of dwell time. Estimates for the $6 \hbar k$ interferometer were based on a contrast measurement at $T=1 \mathrm{~ms}$ and the fairly uniform trend observed with all other LMT orders. (b) Contrast as a function of ARP duration. Uncertainties are smaller than the symbol sizes and lines are guides for the eye.

spontaneous emission-limited contrast for the dwell times, atom cloud size, and Raman pulse parameters considered here. For our pulse parameters, the dynamic phases from $T_{\pi}=3 t_{\pi}$ and $10 t_{\pi}$ ARP pulses vary by 3 and $10 \mathrm{mrad}$, respectively, in response to $0.1 \%$ deviations in optical intensity (i.e., $\Omega_{\text {eff }}$ ). Given our $\mathrm{cm} / \mathrm{s}$-atom velocities and $40-\mu$ s beam splitter pulse spacings, an atom moving in an ideal Gaussian beam experiences pulse-to-pulse intensity variations below $0.1 \%$. Rephasing is highly efficient in this scenario. Therefore, observation of experimental contrast loss indicates that rephasing-though certainly occurring -is limited by beam quality. Exclusive use of optical elements with flatness $\leq \lambda / 10$ should further enhance LMT interferometer contrast.

Estimates of dynamic phase sensitivity to optical intensity (see above) indicate that uncorrelated 100-ppm pulseto-pulse jitter in overall optical power produces an LMT interferometer phase uncertainty of $\sim \sqrt{N} \mathrm{mrad}$. Importantly, this level of power stability is only required during the $\sim 100-\mu$ s beam splitter or mirror sequences, and not over longer times. Experimentally observed phase noise in $6 \hbar k$ interferometers based on tan/tanh and Raman $\pi$ augmentation pulses was similar and largely driven by vibrations, indicating reasonably well-controlled dynamic phase. A more detailed study of phase stability will take place in a vibration-isolated apparatus.

To illustrate the utility of LMT with frequency-swept $\mathrm{ARP}$, recall that the requirements ARP atom optics place on beam quality and optical power control are balanced by the benefits of a higher data rate and atom number, as well as circumvention of evaporative cooling and 2D MOTs. Furthermore, an atom shot-noise-limited accelerometer operating with $10^{6}$ atoms, $T=5 \mathrm{~ms}$, and efficient $22 \hbar k$ ARP beam splitters would resolve $\sim 30 \times 10^{-9}-g$ variations in acceleration per shot. At data rates approaching $100 \mathrm{~Hz}$, such a device would provide $10^{-9}-g / \sqrt{\mathrm{Hz}}$ sensitivity while maintaining sufficient compactness and bandwidth for 
precision inertial sensing. By comparison, a $2 \hbar k$ interferometer would need a dwell time of $T=25 \mathrm{~ms}$ and a corresponding factor-of-5 reduction in data rate and bandwidth to achieve this level of sensitivity.

We have presented cold atom LPAI using atom optics based on stimulated Raman transitions and frequencyswept ARP. By forgoing evaporative cooling and velocity selection, our approach increases the atom number and enables operation at high data rates. These features would be useful for measurement of dynamic signals, such as accelerations and rotations of a moving platform.

This work was sponsored by The C.S. Draper Laboratory. K. K. acknowledges support from the Draper Laboratory Fellowship Program.

[1] A. Peters, K. Y. Chung, and S. Chu, Nature (London) 400 , 849 (1999).

[2] T. L. Gustavson, A. Landragin, and M. A. Kasevich, Classical Quantum Gravity 17, 2385 (2000).

[3] J. B. Fixler, G. T. Foster, J. M. McGuirk, and M. A. Kasevich, Science 315, 74 (2007).

[4] S. W. Chiow, S. Herrmann, S. Chu, and H. Müller, Phys. Rev. Lett. 103, 050402 (2009).

[5] B. Canuel, F. Leduc, D. Holleville, A. Gauguet, J. Fils, A. Virdis, A. Clairon, N. Dimarcq, C. J. Bordé, A. Landragin, and P. Bouyer, Phys. Rev. Lett. 97, 010402 (2006).

[6] R. Geiger, V. Menoret, G. Stern, N. Zahzam, P. Cheinet, B. Battelier, A. Villing, F. Moron, M. Lours, Y. Bidel, A. Bresson, A. Landragin, and P. Bouyer, Nat. Commun. 2, 474 (2011).

[7] A. V. Rakholia, H. J. McGuinness, and G. W. Biedermann, Phys. Rev. Applied 2, 054012 (2014).

[8] S. Dimopoulos, P.W. Graham, J. M. Hogan, M. A. Kasevich, and S. Rajendran, Phys. Rev. D 78, 122002 (2008).

[9] S. Dimopoulos, P. W. Graham, J. M. Hogan, and M. A. Kasevich, Phys. Rev. D 78, 042003 (2008).

[10] P. Storey and C. Cohen-Tannoudji, J. Phys. II (France) 4, 1999 (1994).

[11] J. M. McGuirk, M. J. Snadden, and M. A. Kasevich, Phys. Rev. Lett. 85, 4498 (2000).

[12] A. Sugarbaker, Ph.D. thesis, Stanford University, 2014.
[13] D. L. Butts, K. Kotru, J. M. Kinast, A. M. Radojevic, B. P. Timmons, and R. E. Stoner, J. Opt. Soc. Am. B 30, 922 (2013).

[14] M. Weitz, B. C. Young, and S. Chu, Phys. Rev. Lett. 73, 2563 (1994).

[15] H. Müller, S. W. Chiow, Q. Long, S. Herrmann, and S. Chu, Phys. Rev. Lett. 100, 180405 (2008).

[16] S. W. Chiow, T. Kovachy, H.-C. Chien, and M. A. Kasevich, Phys. Rev. Lett. 107, 130403 (2011).

[17] T. Kovachy, S. W. Chiow, and M. A. Kasevich, Phys. Rev. A 86, 011606 (2012).

[18] P. Clade, S. Guellati-Khélifa, F. Nez, and F. Biraben, Phys. Rev. Lett. 102, 240402 (2009).

[19] H. Müller, S. W. Chiow, S. Herrmann, and S. Chu, Phys. Rev. Lett. 102, 240403 (2009).

[20] H. J. McGuinness, A. V. Rakholia, and G. W. Biedermann, Appl. Phys. Lett. 100, 011106 (2012).

[21] J. Rudolph, W. Herr, C. Grzeschik, T. Sternke, A. Grote, M. Popp, D. Becker, H. Muntinga, H. Ahlers, A. Peters, C. Lammerzahel, K. Sengstock, N. Gaaloul, W. Ertmer, and E. Rasel, arXiv:1105.00403v1.

[22] T. Muller, M. Gilowski, M. Zaiser, P. Berg, C. Schubert, T. Wendrich, W. Ertmer, and E. M. Rasel, Eur. Phys. J. D 53, 273 (2009).

[23] B. Young, M. Kasevich, and S. Chu, in Atom Interferometry, edited by P. R. Berman (Academic Press, San Diego, 1997) pp. 363-406.

[24] K. Kotru, J. M. Brown, D. L. Butts, J. M. Kinast, and R. E. Stoner, Phys. Rev. A 90, 053611 (2014).

[25] A. Abragam, Principles of Nuclear Magnetic Resonance (Clarendon Press, Oxford, 1996).

[26] J. Bateman and T. Freegarde, Phys. Rev. A 76, 013416 (2007).

[27] B. W. Shore, K. Bergmann, A. Kuhn, S. Schiemann, J. Oreg, and J. H. Eberly, Phys. Rev. A 45, 5297 (1992).

[28] J. Baum, R. Tycko, and A. Pines, Phys. Rev. A 32, 3435 (1985).

[29] T.-L. Hwang, P. C. M. van Zijl, and M. Garwood, J. Magn. Reson. 133, 200 (1998).

[30] M. Bason, M. Viteau, N. Malossi, P. Huillery, E. Arimondo, D. Ciampini, R. Fazio, V. Giovannetti, R. Mannella, and O. Morsch, Nat. Phys. 8, 147 (2012).

[31] B. Everitt and D. J. Hand, Finite Mixture Distributions, Monographs on Applied Probability and Statistics (Chapman and Hall, London, 1981).

[32] R. Stoner, D. Butts, J. Kinast, and B. Timmons, J. Opt. Soc. Am. B 28, 2418 (2011). 